\title{
Características produtivas e conservação pós-colheita de cebola em dife- rentes espaçamentos de plantio
}

\author{
Geraldo M. de Resende; Nivaldo Duarte Costa \\ Embrapa Semi-Árido, C. Postal 23, 56300-970 Petrolina-PE; E-mail: gmilanez@cpatsa.embrapa.br.
}

\begin{abstract}
RESUMO
O efeito de diferentes espaçamentos entrelinhas e entre plantas, foi avaliado nas características produtivas e na conservação póscolheita de bulbos de cebola. O experimento foi conduzido de abril a novembro de 1998, na Embrapa Semi-Árido, em Petrolina (PE). O delineamento experimental utilizado foi de blocos ao acaso, no esquema fatorial $2 \times 3$, compreendendo dois espaçamentos entrelinhas $(0,10$ e $0,15 \mathrm{~m})$ e três espaçamentos entre plantas $(0,10 ; 0,20$ e 0,30 $\mathrm{m})$, sendo utilizada a cultivar Franciscana IPA-10 com quatro repetições. $\mathrm{O}$ espaçamento entrelinhas com $0,10 \mathrm{~m}$ alcançou maior produtividade comercial (39,30 t/ha) comparativamente a $0,15 \mathrm{~m}(37,67$ t/ha). Ao se analisar o espaçamento entre plantas verificou-se uma redução linear com o aumento do espaçamento, tendo o espaçamento com $0,10 \mathrm{~m}$ propiciado o maior rendimento com $42,88 \mathrm{t} / \mathrm{ha}$. O menor rendimento de bulbos não comerciais foi proporcionado pelos espaçamentos de 0,29 e $0,26 \mathrm{~m}$ entre plantas, respectivamente, para os espaçamentos entrelinhas de 0,10 e $0,15 \mathrm{~m}$. O peso fresco dos bulbos aumentou linearmente à medida que se aumentaram os espaçamentos entre plantas. Maior porcentagem de bulbos pequenos e médios foi verificada nos menores espaçamentos. Entretanto, à medida que se incrementou o espaçamento entre plantas ocorreu maior perda de peso pós-colheita.
\end{abstract}

Palavras-chave: Allium cepa, rendimento, densidade de plantio, perda de peso de bulbos.

\begin{abstract}
Yield characteristics and postharvest conservation of onion under different planting spacing

The effect of different planting spacing was observed on the yield characteristics and the postharvest conservation of onion bulbs. The experiment was carried out from April to November/1998, in Petrolina, Pernambuco State, Brazil. The experimental design was of randomized complete blocks in a $2 \times 3$ factorial scheme, with four replications. The cultivar Franciscana IPA-10 was planted in 0.10 and $0.15 \mathrm{~m}$ between row spacing and in $0.10 ; 0.15$ and $0.30 \mathrm{~m}$ within row spacing. Highest marketable yield (39.30 t/ha) was obtained form $0.10 \mathrm{~m}$ between row spacing as compared to the row spacing of $0.15 \mathrm{~m}(37.67 \mathrm{t} / \mathrm{ha})$. A negative linear effect for yield was verified, when increasing the within-row spacing, with the $0.10 \mathrm{~m}$ within row spacing showing the highest yield (42.88 t/ha). The smallest yield of unmarketable bulbs (refuses) was observed for 0.29 and $0.26 \mathrm{~m}$ within-row spacing, respectively, for the row spacings of 0.10 and $0.15 \mathrm{~m}$. The bulb fresh weight increased linearly when increasing the spacing inside the row. Larger percentage of small and medium bulbs was observed in the smallest spacings. After harvest, greater reduction in bulb weight was observed when increasing the within-row plant spacing.
\end{abstract}

Keywords: Allium cepa, yield, fresh weight of bulb, planting density, weight loss of bulbs.

\section{(Recebido para publicação em 20 de fevereiro de 2004 e aceito em 8 de abril de 2005)}

A produção mundial de cebola (Allium cepa L.), em 2002, foi de 50,36 milhões de toneladas, tendo sido cultivada em uma área 2,95 milhões de hectares, com produtividade média de 17,07 t/ha (FAO, 2003). No Brasil, a cebola ocupa o terceiro lugar em importância econômica (SOUZA; RESENDE, 2002). A produtividade média nacional é de 17,53 t/ha, sendo que nos estados de Pernambuco e Bahia, maiores produtores do Nordeste, obteve-se a produtividade média de 18,07 e 24,09 t/ha, respectivamente (IBGE, 2003).

A população de plantas ideal é aquela suficiente para atingir o índice de área foliar (IAF) ótimo, a fim de interceptar o máximo de radiação solar útil à fotossíntese e ao mesmo tempo maximizar a fração da massa seca alocada. A população de plantas afeta a penetração da radiação solar no dossel e o equilíbrio entre o crescimento das partes vegetativas e produtivas (MARCELIS, 1993). Modificações na eficiência das fontes, pela alteração na população de plantas ou pelo aumento da disponibilidade de radiação (HAO; PAPADOPOULOS, 1999), afetam indiretamente a distribuição da massa seca entre os órgãos da planta (MARCELIS, 1993).

Em plantas conduzidas sob condições ideais, a bulbificação é acelerada com alta densidade de plantas (RABINOWITCH; BREWSTER, 1990). Em casa de vegetação, Herison et al. (1993), avaliando três densidades de semeio, em bandejas de plástico com 200 células (uma, duas e três plantas por célula) verificaram que as células com duas e três mudas produziram bulbos com menor diâmetro $(76 \mathrm{~mm})$ quando comparados com uma única planta, que produziu maior porcentagem de bulbos de diâmetro superior a $102 \mathrm{~mm}$. Estudando os espaçamentos de 10x15 e 20x15 cm, Viegas D’Abreu (1996), verificou que a maior produtividade foi obtida no menor espaçamento e que o maior peso fresco do bulbo $(145,7 \mathrm{~g} /$ bulbo) foi verificado no maior espaçamento quando comparado ao peso de $118 \mathrm{~g} /$ bulbo obtido no espaçamento de 10x15 cm.

A produtividade comercial aumentou e o peso fresco do bulbo diminuiu quando o número de linhas por canteiro passou de duas para quatro e o espaçamento entre plantas diminui de 21,9 para 7,6 cm. A maior porcentagem de bulbos pequenos e médios foi verificada nos menores espaçamentos (STOFFELLA, 1996). Galmarini e Gaspera (1995) observaram aumento na produtividade da cebola com o incremento da densidade, sendo de 29.921 e 
$62.864 \mathrm{~kg} / \mathrm{ha}$, respectivamente, para 156.000 e 830.000 plantas/ha. Todavia, o tamanho do bulbo diminuiu e os autores recomendaram densidades entre 300.000 a 415.000 plantas/ha como as ideais. Boff et al. (1998), avaliando a influência da densidade de plantas sobre a produtividade e incidência de doenças em três espaçamentos $(10 \times 20 \mathrm{~cm} ; 8 \times 40 \mathrm{~cm}$ e $10 \times 50 \mathrm{~cm}$ ), constataram que o menor espaçamento $(10 \times 20 \mathrm{~cm})$ apresentou a maior produtividade total e comercial e menor peso fresco do bulbo.

$\mathrm{O}$ incremento na densidade de plantio proporcionando aumento na produtividade total e redução do tamanho do bulbo é também relatado por outros autores (WILLIAMS et al., 1973; BREWESTER; SALTER, 1980; HARTRIDGE-ESH; BENNET, 1980; MCGEARY, 1985). Por outro lado, Sabota e Downes (1981) não observaram diferenças significativas na produtividade quando compararam duas populações de plantas (192.940 e 257.320 plantas/ ha), na cultivar Texas Grano. Da mesma forma, avaliando duas densidades de plantio $(7,5 \mathrm{~cm}$ entre plantas e $12,5 \mathrm{e}$ 15,0 cm entrelinhas), Aujla e Madan (1992) não encontraram diferenças na produtividade e na altura da planta. Entretanto, o maior espaçamento aumentou o número de folhas e o diâmetro transversal do bulbo.

O objetivo deste estudo foi avaliar o efeito de diferentes espaçamentos sobre as características produtivas e a conservação pós-colheita dos bulbos de cebola cultivar Franciscana IPA-10, nas condições do Vale do São Francisco no período de inverno.

\section{MATERIAL E MÉTODOS}

O experimento foi conduzido de abril a novembro de 1998, na Embrapa SemiÁrido, em Petrolina, a 9 $24^{\prime}$ de latitude Sul e $40^{\circ} 29^{\prime}$ de longitude Oeste, e 365,5 $\mathrm{m}$ de altitude. $\mathrm{O}$ solo classificado como Latossolo Vermelho Amarelo Distrófico, apresentou $\mathrm{pH}\left(\mathrm{H}_{2} \mathrm{O}\right)=6,2 ; \mathrm{Ca}=2,2$ $\mathrm{cmol}_{\mathrm{c}} / \mathrm{dm}^{3} ; \mathrm{Mg}=0,7 \mathrm{cmol}_{\mathrm{c}} / \mathrm{dm}^{3} ; \mathrm{Na}=$ $0,01 \mathrm{cmol} / \mathrm{dm}^{3} ; \mathrm{K}=0,27 \mathrm{cmol} / \mathrm{dm}^{3} ; \mathrm{Al}$ $=0,05 \mathrm{cmol} / \mathrm{dm}^{3}, \mathrm{P}($ Mehlich $) \stackrel{c}{=} 7,0 \mathrm{mg} /$ $\mathrm{dm}^{-3}$ e M.O. $=6,8 \mathrm{~g} / \mathrm{kg}$.

$\mathrm{O}$ delineamento experimental utilizado foi blocos ao acaso, em esquema fatorial $2 \times 3$, compreendendo dois espaçamentos entrelinhas $(0,10$ e 0,15 m) e três espaçamentos entre plantas $(0,10 ; 0,20$ e $0,30 \mathrm{~m})$ com quatro repetições. A unidade experimental constituiu-se de um canteiro 3,0 m de comprimento por $1,2 \mathrm{~m}$ de largura, sendo usado como área útil 1,8 m² (3,0x0,6 m). A adubação de plantio constou de 600 $\mathrm{kg} / \mathrm{ha}$ da fórmula NPK 6-24-12, baseada na análise do solo. Foram aplicados em cobertura $200 \mathrm{~kg} / \mathrm{ha}$ de uréia e 50 $\mathrm{kg} / \mathrm{ha}$ de cloreto de potássio, parcelados aos 15 e 30 dias após o transplante.

A semeadura da cultivar Franciscana IPA-10 foi feita em 17/04/1998 e o transplante efetuado 30 dias após. O preparo do solo constou de aração, gradagem e levantamento dos canteiros a 0,20 m de altura. A cultura foi mantida no limpo através de capinas manuais e a irrigação por microaspersão realizada três vezes por semana, com lâminas em torno de $10 \mathrm{~mm}$, baseada na evaporação do tanque classe A, e suspensas 20 dias antes da colheita. Os demais tratos fitossanitários foram realizados de acordo com as recomendações para a cultura da cebola.

A colheita foi realizada em 04/09/ 1998 quando as plantas apresentaram sinais avançados de senescência, como amarelecimento e seca das folhas e quando mais de $70 \%$ das plantas encontravam-se estaladas. A cura foi realizada ao sol por três dias e 12 dias à sombra em galpão ventilado.

Foram avaliadas a produtividade comercial de bulbos (bulbos perfeitos, livres de pragas e doenças, sem danos mecânicos e com diâmetro transversal acima de $35 \mathrm{~mm}$ ), expressa em t/ha, aos 15 dias após a cura. Refugos foram considerados os bulbos charutos, podres, com danos mecânicos e com diâmetro inferior a $35 \mathrm{~mm}$. A massa fresca de bulbo ( $\mathrm{g} / \mathrm{bulbo}$ ) foi determinada dividindo-se o peso de bulbos comerciais após a cura pelo número de bulbos. Após o período de cura, os bulbos foram armazenados à temperatura ambiente e realizadas pesagens aos 20; 40 e 60 dias, sendo os valores comparados àqueles obtidos ao final da cura (15 dias após colheita). Os valores foram transformados em porcentagem de perda de peso. A classificação de bulbos comerciais segundo o diâmetro transversal ( $\mathrm{mm}$ ) foi feita de acordo com Brasil (1995) em Classe 2: maior que 35 até $50 \mathrm{~mm}$ de diâmetro; Classe 3: maior que 50 até 70 $\mathrm{mm}$; Classe 4: maior que 70 ate $90 \mathrm{~mm}$ e Classe 5: maior que $90 \mathrm{~mm}$. Os dados foram expressos em porcentagem.

Segundo a metodologia descrita por Pimentel Gomes (2000), os dados coletados foram submetidos à análise de variância e as médias comparadas pelo teste de Tukey, ao nível de 5\% de probabilidade, e regressão polinomial. Os dados de porcentagem foram transformados em arco-seno $\sqrt{P / 100}$ para efeitos de análise, sendo apresentados nos resultados as médias originais.

\section{RESULTADOS E DISCUSSÃO}

A produtividade comercial de bulbos foi influenciada pelos fatores, sem haver interação dos mesmos. O espaçamento de $0,10 \mathrm{~m}$ entrelinhas apresentou maior produtividade (39,30 t/ha) comparativamente ao espaçamento de 0,15 m (37,67 t/ha). Verificou-se redução linear com o aumento do espaçamento entre plantas, tendo no espaçamento de $0,10 \mathrm{~m}$ entre plantas resultado em maior rendimento com 42,88 t/ha (Figura 1). Estes resultados confirmam as observações de que as cebolas respondem especialmente à variação no espaçamento entre plantas (FILGUEIRA, 1982; MELO et al., 1988). Portanto, pode-se concluir que a produtividade da cebola aumentou à medida que se diminuiu à distância entrelinhas e entre plantas, fato relatado por diversos autores em diferentes épocas (MANGUAL-CRESPO et al., 1979; LOPES, 1987; STOFFELLA, 1996 E BOFF et al., 1998).

Bulbos charutos, podres, ou com danos mecânicos não foram observados no que se refere à produção de refugos (bulbos não comerciais), havendo somente bulbos com diâmetro inferior a $35 \mathrm{~mm}$. Pela derivada das equações de regressão constataram-se que o menor rendimento de bulbos não comerciais foi proporcionado pelos espaçamentos de 0,29 e 0,26 m entre plantas, respectivamente, para os espaçamentos de 0,10 e 0,15 m entrelinhas (Figura 2). 
Detectou-se, com o aumento do espaçamento entre plantas, uma redução gradativa da produção de bulbos considerados não comerciais. Estes resultados mostram relação inversa entre o aumento ou redução do espaçamento entrelinhas e entre plantas na produtividade de bulbos de cebola, ou seja, a variação do número de plantas por unidade de área afeta a produtividade e a qualidade dos bulbos. Em baixas populações se produz, geralmente, baixos rendimentos e alta porcentagem de bulbos médios e grandes. Em cultivos com densidades maiores que a ótima, se têm bulbos pequenos e desuniformes de menor qualidade comercial, comparativamente ao cultivo em densidade adequada.

Notou-se para peso fresco do bulbo um efeito significativo da interação dos fatores. O peso fresco do bulbo aumentou linearmente à medida que se aumentaram os espaçamentos entre plantas. Este incremento foi da ordem de 76,3\% e $71,2 \%$, respectivamente, para os espaçamentos de 0,10 e 0,15 m entrelinhas (Figura 3). Salienta-se que o maior peso fresco do bulbo foi obtido tanto com o aumento do espaçamento entre plantas como com o espaçamento entrelinhas, o que, provavelmente, está relacionado à maior área de exploração das raízes e menor competição pelos fatores de produção como água, luz e nutrientes. Lopes (1987) avaliando diversas cultivares observou que com o incremento da densidade ocorreu redução no diâmetro e na massa fresca do bulbo. A diminuição do peso fresco do bulbo com o incremento da densidade de plantio foi também observado por outros autores (GALMARINI; GASPERA, 1995; STOFFELLA, 1996; LIPINSKI et al., 2002).

A classificação de bulbos de cebola foi influenciada pela interação entre os fatores (Tabela 1). No que se refere à classificação de bulbos classe 2, constatou-se para os espaçamentos de 0,10 e 0,15 m entrelinhas efeitos quadráticos, nos quais os espaçamentos de 0,28 e $0,26 \mathrm{~m}$ entre plantas proporcionaram as menores porcentagens. A medida que se aumentou o espaçamento, foi observada redução gradativa na porcentagem deste tipo de bulbo, que são menores comparativamente às demais classes.

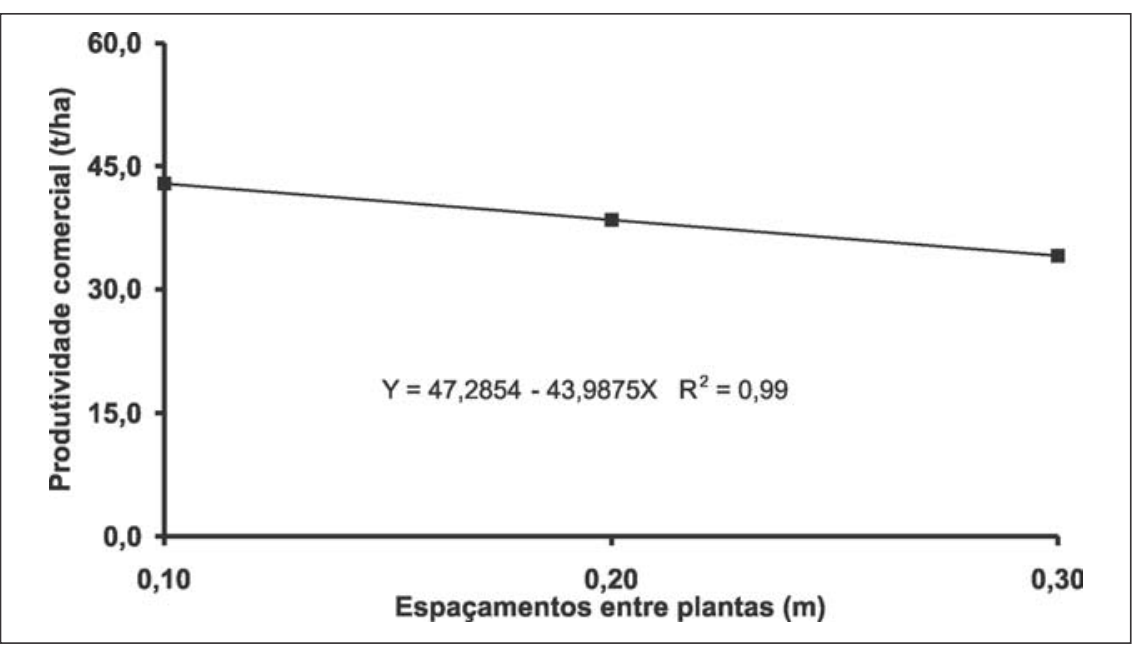

Figura 1. Produtividade comercial de bulbos de cebola, cv. Franciscana IPA-10, em função dos espaçamentos entre plantas. Petrolina, Embrapa Semi-Árido, 1998.

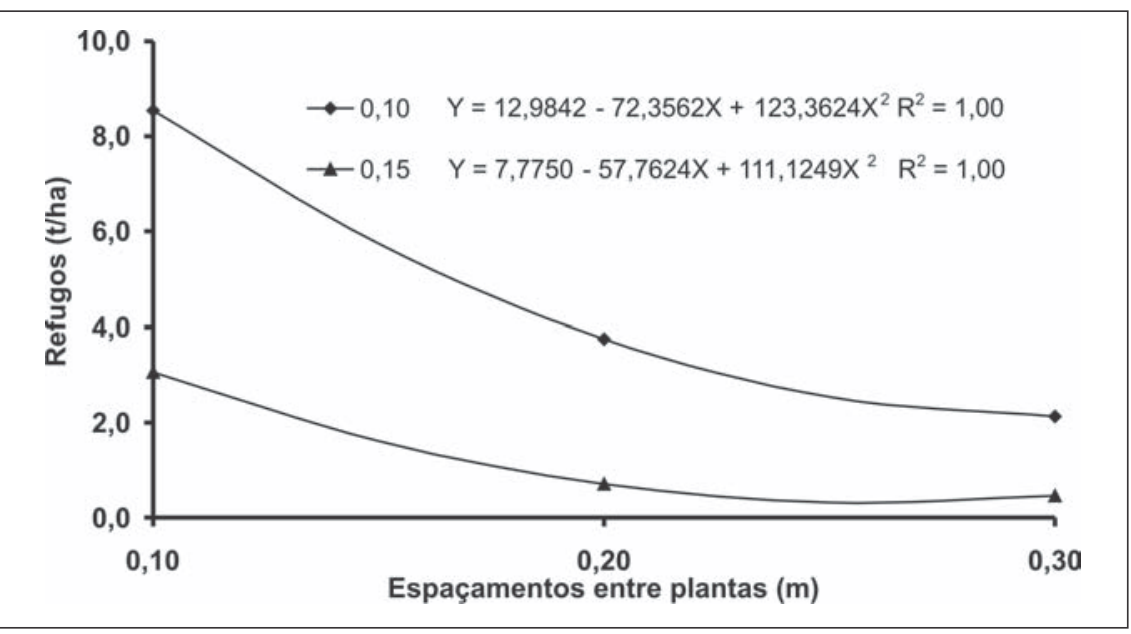

Figura 2. Refugos (produtividade não comercial) de bulbos de cebola, cv. Franciscana IPA10, nos espaçamentos 0,10 e 0,15 m entrelinhas em função dos espaçamentos entre plantas. Petrolina, Embrapa Semi-Árido, 1998.

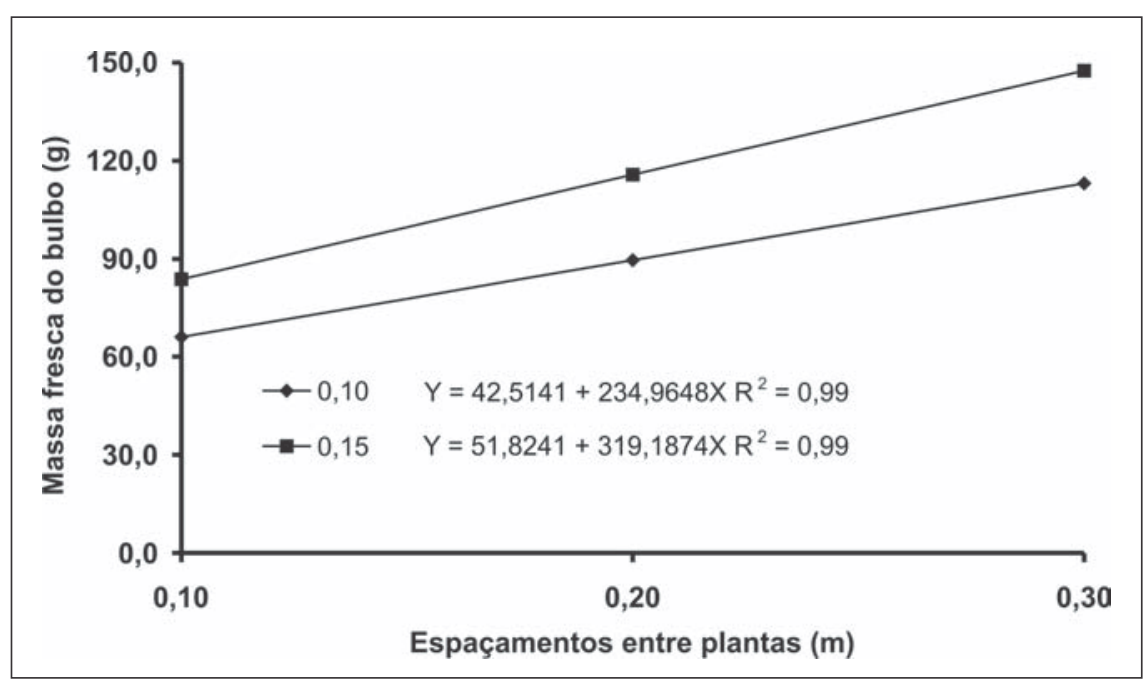

Figura 3. Massa fresca do bulbo de cebola, cv. Franciscana IPA-10, nos espaçamentos 0,10 e 0,15 m entrelinhas em função dos espaçamentos entre plantas. Petrolina, Embrapa SemiÁrido, 1998. 
Tabela 1. Equações de regressão para classificação de bulbos de cebola, cv. Franciscana IPA-10, em classes (\%), segundo o diâmetro transversal, em função dos espaçamentos entre plantas. Petrolina, Embrapa Semi-Árido, 1998.

\begin{tabular}{lll}
\hline Classes & \multicolumn{1}{c}{ Equações de regressão } \\
\hline \multirow{2}{*}{ Classe 2 } & $\mathrm{Y}(0,10 \mathrm{~m})=74,2436-347,3274 X+631,3548^{* *} \mathrm{X}^{2}$ & $\mathrm{R}^{2}=1,00$ \\
& $\mathrm{Y}(0,15 \mathrm{~m})=62,6597-326,2957 \mathrm{X}+636,8676^{* *} \mathrm{X}^{2}$ & $\mathrm{R}^{2}=1,00$ \\
\hline \multirow{2}{*}{ Classe 3 } & $\mathrm{Y}(0,10 \mathrm{~m})=10,9877+399,3718 \mathrm{X}-851,3980^{* *} \mathrm{X}^{2}$ & $\mathrm{R}^{2}=1,00$ \\
& $\mathrm{Y}(0,15 \mathrm{~m})=39,3690+180,1925 \mathrm{X}-581,1522^{* *} \mathrm{X}^{2}$ & $\mathrm{R}^{2}=1,00$ \\
\hline \multirow{2}{*}{ Classe 4 } & $\mathrm{Y}(0,10 \mathrm{~m})=13,1267-60,0451 \mathrm{X}+296,7962^{* *} \mathrm{X}^{2}$ & $\mathrm{R}^{2}=1,00$ \\
& $\mathrm{Y}(0,15 \mathrm{~m})=-3,5535+150,2379^{* *} \mathrm{X}$ & $\mathrm{R}^{2}=0,98$ \\
\hline
\end{tabular}

** Significativo ao nível de $1 \%$ de probabilidade, pelo teste de $\mathrm{F}$.

Para a classe 3 (bulbos de tamanho intermediário), os espaçamentos de 0,23 e $0,16 \mathrm{~m}$ entre plantas promoveram as maiores porcentagens deste tipo de bulbo. Para a classe 4, (bulbos de maior tamanho), registrou-se, para o espaçamento de 0,10 m entrelinhas, menor porcentagem de bulbos no espaçamento de $0,10 \mathrm{~m}$ entre plantas. Este resultado infere ser o menor espaçamento aquele que propicia menor produtividade de bulbos de tamanho superior. Para o espaçamento de $0,15 \mathrm{~m}$ entrelinhas, obteve-se aumento linear com o aumento do espaçamento entrelinhas. Maior número de bulbos grandes foi obtido com o aumento no espaçamento entre plantas (Tabela 1), resultados estes que corroboram os obtidos por Viegas D’Abreu (1996), que obteve maior tamanho de bulbo no maior espaçamento, assim como os obtidos por Stoffella (1996) que verificou maior porcentagem de bulbos pequenos e médios nos menores espaçamentos, e Rumpel e Felczynski (2000) que encontraram redução na produção de bulbos maiores com o incremento da densidade de plantio.

Os resultados obtidos no presente trabalho evidenciaram uma relação inversa entre a densidade de plantio e o tamanho do bulbo, onde maiores densidades proporcionaram menores bulbos, e vice-versa. Segundo Souza e Resende (2002), o mercado consumidor nacional prefere bulbos de tamanho médio com pesos de 80 a 100 gramas e diâmetro transversal de 40 a $80 \mathrm{~mm}$, que se enquadram perfeitamente dentro das faixas de tamanho de bulbo obtidas no presente estudo.

A perda de peso aos 20, 40 e 60 dias após cura (DAC) apresentou efeitos sig- nificativos apenas para o espaçamento entre plantas, não se constatando efeito significativos para o espaçamento entrelinhas e nem para a interação dos fatores. Para porcentagem de perda de peso aos 20 dias $\left(\mathrm{Y}=11,3905+4,2763 \mathrm{X} \mathrm{R}^{2}\right.$ $=0,99)$ e 40 dias após cura $(\mathrm{Y}=13,5502$ $\left.+10,0059 \mathrm{X} \mathrm{R} \mathrm{R}^{2}=0,98\right)$, verificou-se aumentos lineares evidenciando maiores perdas de peso com o incremento dos espaçamentos. Pelos coeficientes angulares das equações de regressão estimase esta perda de peso em $0,43 \%$ e $1,00 \%$ para cada aumento do espaçamento entre plantas. Para a perda de peso aos 60 dias após a cura $(\mathrm{Y}=17,1576$ $\left.15,9001 X+68,87772 X^{2} R^{2}=1,00\right)$ verificou-se menor perda no espaçamento de $0,11 \mathrm{~m}$ entre plantas, a qual foi de $16,14 \%$. Inferiu-se por estes resultados, que a maior perda ocorreu em espaçamento maiores, que determinaram maior tamanho de bulbos, com conseqüente maior teor de água nos bulbos. Calbo et al. (1980) observaram perdas de $30 \%$ a $100 \%$ em bulbos de cebola "Baia Periforme" armazenadas até 70 dias, a granel e réstias em diferentes recipientes, assim como Resende et al. (2004) relatam para a cultivar Texas Grano PRR que o espaçamento de 0,30 $\mathrm{m}$ entre plantas foi o que promoveu maior perda de peso de bulbos de cebola para o espaçamento de $0,15 \mathrm{~m}$ entre linhas $(43,16 \%)$, sob as condições do Vale do São Francisco.

Em função das diferentes características avaliadas, recomenda-se os espaçamentos de 0,10 ou $0,15 \mathrm{~m}$ entrelinhas e $0,10 \mathrm{~m}$ entre plantas como os mais adequados para o cultivo da cebola cultivar Franciscana IPA-10, nas condições do Vale do São Francisco, no período de inverno.

\section{LITERATURA CITADA}

AUJLA, T.S.; MADAN, P.S. Response of onion (Allium cepa) to irrigation, nitrogen fertilizer and row spacing on deep sandy-loam in subtropical monsoon region. Indian Journal of Agricultural Science, New Delhi, v.62, n.2, p.129-134, 1992. BOFF, P., HENRI, S.; GONÇALVES, P.A.S. Influência da densidade de plantas na ocorrência de doenças foliares e produção de bulbos de cebola. Fitopatologia Brasileira, Brasília, v.23, n.4, p.448452, 1998.

BRASIL. Ministério da Agricultura, Abastecimento e Reforma Agrária. Portaria n.529 de 18 ago. 1995. Diário Oficial da República Federativa do Brasil, Brasília, 1 set.1995, Seção 1, p.13513.

BREWESTER, J.L., SALTER, P.J. The effect of plant spacing on the yield and bolting of two cultivars of overwintered bulb onions. Journal of Horticultural Science, Ashford, v.55, n.2, p.97102, 1980

CALBO,A.G.; CORDEIRO, C.M.T.; GUALBERTO, A.G. Efeito do acondicionamento sobre o comportamento fisiológico e conservação de duas cultivares de cebola (Allium cepa) no Vale do São Francisco. Revista de Olericultura, v.18, p.86-99, 1980.

FAO. Agricultural production, primary crops. Disponível em: <http://www.fao.org > Acesso em: 06 mai. 2003.

FILGUEIRA, F.A.R. Manual de Olericultura: cultura e comercialização de hortaliças. 2. ed., São Paulo: Agronômica Ceres, 1982. v.2, 357 p. GALMARINI, C.R.; GASPERA, P.G. Efecto de la epoca de transplante y la densidad de plantacion en el cultivo de cebolla tipo Valenciana. Horticultura Argentina, Mendoza, v.14, n.37. p.23-29, 1995.

HAO, X.; PAPADOPOULOS, A.P. Effects of supplemental lighting and cover materials on growth, photosynthesis, biomass partitioning, early yield and quality of greenhouse cucumber. Scientia Horticulturae, v.80, n.1-2, p.1-18, 1999.

HARTRIDGE-ESH, K.A.; BENNET, J.P. Effects of seed weight plant density and spacing on yield response of onion. Journal of Horticultural Science, Ashford, v.55, n.3, p.247-252, 1980. HERISON, C.; MASABNI, J.G.; ZANDSTRA, B.H. Increasing seedling density, age, and nitrogen fertilization increases onion yield. HortScience, Alexandria, v.28, n.1, p.23-25, 1993.

INSTITUTO BRASILEIRO DE GEOGRAFIA E ESTATÍSTICA - IBGE. Levantamento sistemático da produção agrícola. Rio de Janeiro: v. 15 n.03 p.1-79, 2003.

LIPINSKI, V.M., GAVIOLA, S.; GAVIOLA, J.C. Efecto de la densidad de plantación sobre el rendimiento de cebolla cv. Cobriza Inta con riego por goteo. Agricultura Técnica, Santiago, v.62,n.4, p.574-582, 2002.

LOPES, J.F. Effects of planting dates and spacing on several characteristics of short day onion varieties grown in South Texas. 1987. 111 f. (Tese Doutorado) Texas University.

MANGUAL-CRESPO, G.; RAMIREZ, C.T.; ORENGO, E. Effect of plant spacing and fertlizer levels on yield and dry bulb weight of onion cv. Texas Grano 502. Journal of Agriculture of the University of Puerto Rico, Rio Piedras, v.63, n.4, p.417-422, 1979. 
MARCELIS, L.F.M. Fruit growth and biomass allocation to the fruits in cucumber. 2. Effect of irradiance. Scientia Horticulturae, v.54, n.2, p.123130, 1993.

MCGEARY, D.F. The effect of plant density on shape, size, uniformity, soluble solids content and yield of onions suitable for pickling. Journal Horticultural Science, Ashford, v.60, n.1, p.83-87, 1985.

MELO, P.C.T.; RIBEIRO, A.; CHURATA-MAS-

CA, M.G.C. Sistemas de produção, cultivares de cebola e seu desenvolvimento para as condições brasileiras. In: SEMINÁRIO NACIONAL DE CEBOLA, 3, 1988, Piedade-SP. Anais.. Jaboticabal: FUNEP, 1988. p.27-61.

PIMENTEL GOMES, F. Curso de estatística experimental. 14.ed. São Paulo: Nobel, 2000. 477 p.

RABINOWITCH, H.D.; BREWSTER, J.L. Onions and Allied Crops. Boca Raton: CRC Press, 1990. 273 p.
RESENDE, G.M.; COSTA, N.D.; ALVARENGA, M.A.R. Características produtivas e conservação pós-colheita da cebola (Allium Cepa L.) cv. Texas Grano PRR em diferentes espaçamentos de plan-

tio. Horticultura Brasileira, Brasília, v.22, n.2, julho 2004, Suplemento 2. CD-ROM. Trabalho apresentado no $44^{\circ}$ Congresso Brasileiro de Olericultura, 2004.

RUMPEL, J.; FELCZYNSKI, K. Effect of plant density on yield and bulb size of direct sown onions. Acta Horticulturae, Bari, n.533, p.179186, 2000.

SABOTA, C.M.; DOWNES, J.D. Onion growth na yield in relation to transplant, pruning, size, spacing and depth of planting. Hortscience, Alexandria, v.16, n.4, p.533-535, 1981.

SOUZA, R.J., RESENDE, G.M. Cultura da cebola. 2002.115 f. (Textos Acadêmicos) Universidade Federal de Lavras, Lavras.
STOFFELLA, P.J. Planting arrangement and density of transplants influence sweet Spanish onion yields and bulb size. Hortscience, Alexandria, v.31, n.7, p.1129-1130, 1996.

VIEGAS D'ABREU, D.A. Effect of nitrogen and spacing on bulb splitting in onion cv. Bombay Red. TVIS Newsletter, Luanda, v.1, n.2, p.26, 1996.

WILLIAMS, C.F.; CARVIN, C.; MACK, H.J.; LAWS, W.D. Effect of spacing on weed competition in sweet corn, snap beans and onions. Journal of the American Society Horticultural Science, St. Joseph, v.98, n.6, p.526-29, 1973. 